\title{
Las competencias transversales en el TFG. Valoración en el Grado de Biotecnología
}

Natalia Lajara-Camilleria, Andrés Rovira Cardete ${ }^{b}$, Alexis Bañón Gomis ${ }^{c}$, Juan A. Marín-García

Universitat Politècnica de València

analade@cegea.upv.es banrocar@mcm.upv.es, calbaogo@upvnet.upv.es, d jamarin@omp.upv.es

\begin{abstract}
The necessary integration and accreditation of cross-curricular skills (CTs in Spanish) in the Bachelor and Master level has revealed the need to analyze in detail when, how and by whom are worked each of these skills throughout the degree.

The student can achieve an appropiate level by developing he competence through regular courses, the final degree thesis (TFG in Spanish) or by complmentary activities.

The aim of this study is to determine ehich of the CTs defined in the UPV for graduates can be effectively developed and evaluated through the TFG in the degree of Biotechnology. To this end, a Delphi study has been developed among lecturers of this degree.

After two rounds of opinión the grid of the most relevant CTs for biotechnologists has been obtained, as well as a proposal of an evaluation matrix for each of the CTs, including agents.

Results show the fundamental role that the students plays in this process, as well as their director.
\end{abstract}

Keywords: EEES, competency assessment, UPV cross-curricular competencies, TFG, Delphi

\section{Resumen}

La necesaria incorporación y acreditación de las competencias transversales (CTs) en las titulaciones de grado y máster ha puesto de manifiesto la 
Las competencias transversales en el TFG. Valoración en el Grado de Biotecnología

necesidad de analizar con detenimiento cuándo, cómo y por quién son trabajadas cada una de las competencias a lo largo de la titulación.

El estudiante puede alcanzar un nivel competencial adecuado mediante el desarrollo a través de asignaturas, a través del trabajo final de grado (TFG) o bien por actividades complementarias.

El objetivo de este trabajo es determinar cuáles de las CTs definidas en la UPV para sus egresados pueden ser efectivamente desarrolladas y evaluadas a través del TFG en el Grado de Biotecnología. Para ello se ha realizado una investigación siguiendo el método Delphi seleccionando como expertos participantes a 28 profesores que tienen docencia en la titulación.

Tras dos rondas de opinión se ha obtenido el mapa de competencias transversales que resultan más relevantes para el profesional de Biotecnología y se ha propuesto una matriz de evaluación por agentes (estudiante, tutor del TFG, tribunal y comisión) para cada una de las CTs.

Los resultados muestran el papel fundamental que desempeña no sólo el estudiante sino también el tutor en la evaluación de las CTs en el TFG.

Palabras clave: EEES, evaluación de competencias, competencias transversales UPV, TFG, Delphi

\section{Introducción}

Las universidades españolas han asistido en la última década a una intensa serie de cambios que han tenido como consecuencia una reconversión profunda de su oferta. No es posible acometer esta empresa sin asumir importantes retos por parte de todos los estamentos de la comunidad universitaria

Uno de los desafíos que se presentan a corto plazo en la Universitat Politècnica de València (UPV) consiste en implementar con éxito las llamadas "competencias transversales UPV" (CTs). Se pretende sintetizar el perfil competencial que adquieren todos los alumnos de las titulaciones de la UPV en una serie de 13 competencias, que son las que siguen (UPV, 2013):

1 Comprensión e integración.

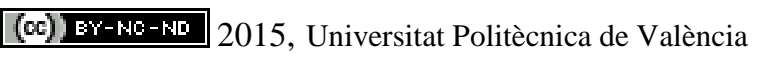

Congreso IN-RED (2015) 
2 Aplicación y pensamiento práctico.

3 Análisis y resolución de problemas.

4 Innovación, creatividad y emprendimiento.

5 Diseño y proyecto.

6 Trabajo en equipo y liderazgo.

7 Responsabilidad ética, medioambiental y profesional.

8 Comunicación efectiva.

9 Pensamiento crítico.

10 Conocimiento de problemas contemporáneos.

11 Aprendizaje permanente.

12 Planificación y gestión del tiempo.

13 Instrumental específica.

La adquisición y evauación de estas competencias se plantea desde tres vías posibles:

- vcurricular, a través de las asignaturas que componen la titulación

- a través del Trabajo Final de Grado

- extracurricular, mediante actividades complementarias que permitan certificar la adquisición de la competencia.

El objetivo de este trabajo aborda la posibilidades de la segunda vía como herramienta de evaluación de las competencias transversales en el Grado en Biotecnología.

Con este fin se presentará la metodología empleada en el estudio, a continuación se exponen los resultados obtenidos y finalmente se incluyen las conclusiones correspondientes.

\section{Metodología}

La metodología utilizada para dar cumplimiento al objetivo del trabajo ha sido una consulta Delphi a expertos. Se ha optado por éste método puesto que cumple con las restricciones que imponen las premisas bajo las que se desea desarrollar el estudio. Los supuestos de partida en los que nos hemos basado son los siguientes:

- El TFG es una herramienta inestimable para desarrollar y cuantificar la adquisición de CTs por parte del estudiante 
Las competencias transversales en el TFG. Valoración en el Grado de Biotecnología

- A priori no podemos asumir que los TFG de todas las titulaciones puedan desarrollar y evaluar las mismas CTs

- Los profesores que actualmente tienen responsabilidades docentes en cada titulación son los más adecuados para determinar los resultados que pretendemos alcanzar ya que ellos son los en principio pueden contar con la información necesaria para evaluar, en el caso concreto de cada Grado, el nivel de idoneidad del TFG como herramienta de evaluación de cada una de las CTs.

A la luz de estas cuestiones la metodología Delphi se configura como la idónea para llevar a cabo el estudio por las razones que se exponen en el subapartado siguiente.

\subsection{El método Delphi como herramienta}

Tal y como hemos señalado, para la realización de este estudio hemos recurrido a la metodología Delphi, al tratarse de una técnica de análisis especialmente útil en aquellos en los que se quiere obtener la opinión sobre un tema concreto del que no existe información previa (Gupta y Clarke, 1996). El punto de partida para el desarrollo de la técnica Delphi es la identificación de los objetivos a estudiar, mediante la formulación de una serie de hipótesis de trabajo, que deben plantear un problema o conjunto de problemas susceptibles de ser tratados por medio de esta metodología (Delbecq, 1989; en Gallego y Juliá, 2003).

La idea que subyace en los planteamientos del presente estudio tiene su origen en la necesidad de armonizar posturas entre los docentes ya que es, con seguridad, un tema de controversia entre los profesores.

Así pues, la opinión del grupo de expertos, procesada mediante la técnica Delphi, permite aunar las diferentes perspectivas de todos los participantes, con perfiles distintos, sobre la materia en cuestión (Landeta, 1999). Cada experto consultado debe pronunciarse en primer lugar sobre cree que cada una de las CTs puede ser evaluada o no a través del TFG en el Grado en biotecnología. En caso de contester afirmativa, se pide especificar quien/es son los agentes que pueden llevar a cabo dicha evaluación (alumno, tutor, tribunal, commission de TFG).

Por ello, el juicio integrado de un grupo de personas dotadas de un elevado nivel de capacidad crítica, puede realizar una mejor aproximación a las necesidades reales en el ámbito cooperativo en materia de formación con mayor fidelidad que cualquier estimación individual (Gallego y Juliá, 2003).

Tras sucesivas rondas de contrastación, las contestaciones individuales son integradas en una respuesta de grupo conjunta. 
Las características definitorias del método Delphi, que lo configuran como una técnica de grupo con personalidad y aplicaciones propias, pueden consultarse, entre otros, en Landeta (1999).

\subsection{Muestra}

El cuestionario elaborado (Anexo I) fue remitido a todos los responsables de asignatura y algunos profesores con docencia en el grado de Biotecnología. De un total de 42 cuestionarios enviados, se obtuvieron 28 respuestas, es decir, una tasa de respuesta del $66,7 \%$.

El cuestionario fue circulado a través de correo electrónico en las dos rondas de preguntas que se llevaron a cabo. En la primera ronda se utilizó la herramienta "formulario" de Google Docs para recoger las respuestas vía web. Esta ronda de consulta se inició en noviembre de 2014. La segunda ronda implica la personalización de la información remitida a cada participante en el estudio puesto que se incorpora no sólo la respuesta alcanzada por el grupo sino también la que aportó el participante en la ronda anterior. Esta fase se llevó a cabo en enero de 2015.

\section{Resultados}

Asumiendo que todas las CTs definidas son deseables en un profesional, sea cual sea su área de trabajo, sí que es posible que existan diferencias en la importancia percibida por parte de los profesores en las distintas CTs para los egresados de esa titulación. Con el animo de poder establecer estas diferencias mediante la comparación con réplicas de este estudio que puedan realizarse posteriormente en otras titulaciones, se consultó esta cuestión a los participantes.

Los resultados obtenidos (Fig.1) muestran que todas las CTs obtienen por término medio puntuaciones superiores a 3 (calificada como término medio). Sin embargo se aprecian diferencias significativas entre la CT considerada como más relevante, CT1- Comprensión e integración, y la que obtiene una puntuación más baja, la CT4- Creatividad, innovación y emprendimiento. 
Las competencias transversales en el TFG. Valoración en el Grado de Biotecnología

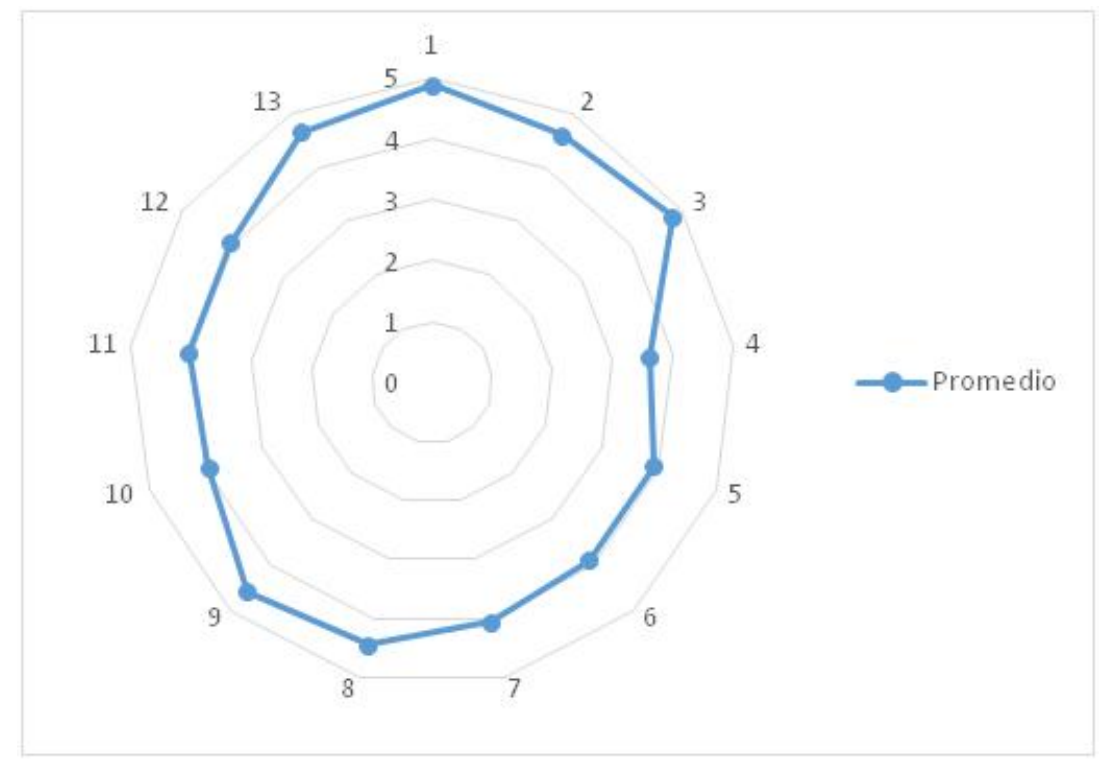

Fig. 1 Valoración de la relevancia de las CTs para los graduados en Biotecnología

En referencia a la cuestión central de este trabajo, sobre la factibilidad de evaluar las CTs en el TFG en el Grado de Biotecnología, y, en ese caso, quién/es sería/n los posibles responsables de llevar a cabo dicha evaluación, los resultados obtenidos (Tabla 1) muestran cierto consenso en algunas de las competencias si bien no se ha podido lograr un acuerdo en todas ellas ya que en la segunda ronda se registró una gran estabilidad respecto a las respuestas de la primera ronda, sin que mejorara el consenso alcanzado.

Tabla 1. Valoración de la factibilidad de evaluar las CTs en el TFG de Biotecnología

\begin{tabular}{|c|c|c|c|c|c|c|}
\hline & Competencia & $\begin{array}{l}1 \text { No se } \\
\text { puede }\end{array}$ & 2 Tutor & $\begin{array}{c}3 \\
\text { Comisió } \\
\mathbf{n}\end{array}$ & 4 Tribunal & 5 Alumno \\
\hline 1 & $\begin{array}{c}\text { Comprensión e } \\
\text { integración }\end{array}$ & & SÍ & & SÍ & \\
\hline 2 & $\begin{array}{c}\text { Aplicación y } \\
\text { pensamiento } \\
\text { práctico }\end{array}$ & & Sí & & & \\
\hline 3 & Análisis y & & SÍ & & ? & \\
\hline
\end{tabular}




\begin{tabular}{|c|c|c|c|c|c|}
\hline & $\begin{array}{l}\text { resolución de } \\
\text { problemas }\end{array}$ & & & & \\
\hline 4 & $\begin{array}{l}\text { Innovación, } \\
\text { creatividad y } \\
\text { empr. }\end{array}$ & SÍ & SÍ & & \\
\hline 5 & Diseño y proyecto & SÍ & sí & & \\
\hline 6 & $\begin{array}{l}\text { Trabajo en equipo } \\
\text { y liderazgo }\end{array}$ & SÍ & sí & & $?$ \\
\hline 7 & $\begin{array}{l}\text { Resp. ética, medio. } \\
\text { y profesional }\end{array}$ & SÍ & SÍ & & $?$ \\
\hline 8 & $\begin{array}{l}\text { Comunicación } \\
\text { efectiva }\end{array}$ & & SÍ & SÍ & \\
\hline 9 & $\begin{array}{l}\text { Pensamiento } \\
\text { crítico }\end{array}$ & & SÍ & SÍ & \\
\hline 10 & $\begin{array}{c}\text { Planificación y } \\
\text { gestión del tiempo }\end{array}$ & & SÍ & & sí \\
\hline 11 & $\begin{array}{l}\text { Aprendizaje } \\
\text { permanente }\end{array}$ & SÍ & $?$ & & Sí \\
\hline 12 & $\begin{array}{c}\text { Conocimiento de } \\
\text { problemas... }\end{array}$ & SÍ & & SÍ & \\
\hline 13 & $\begin{array}{l}\text { Instrumental } \\
\text { específica }\end{array}$ & & Sí & Sí & ? \\
\hline
\end{tabular}

Se marca Sí cuando esta respuesta ha sido marcada por más de un 30\% e ? cuando ha sido marcada entre 20 y $30 \%$ de las respuestas.

Es relevante destacar también el rol fundamental que se asigna tanto al alumno como al tutor en la evaluación de las CTs a trave's del TFG. El tutor debe participar en la evaluación de al menos 11 de las 13 CTs definidas, al tiempo que el alumno es quien se estima que mejor puede evaluar las cuestiones referentes a planificación y gestión del tiempo y aprendizaje permanente.

\section{Conclusiones}

Las CTs son un reto al que se enfrenta la UPV a corto plazo, que va a suponer la necesidad de determinar la forma de desarrollar, evaluar y acreditar la consecución de ciertas habilidades por parte de los egresados. 
Una de las vías planteadas para esta tarea es la del TFG. En este trabajo se plantea un estudio de carácter exploratorio con el fin de determinar la opinión de una muestra de profesores del Grado en Biotecnología. Cabe tener en cuenta que no se ha conseguido el consenso en las cuestiones planteadas por lo que los resultados obtenidos deben ser interpretados con cautela.

Sin embargo sí que es posible afirmar que los resultados muestran una elevada heterogeneidad en las opiniones, mayor de la que cabría esperar, lo cual puede deberse en parte a una falta de comprensión clara del concepto de cada una de las dimensiones. No obstante, sí que puede concluir que una mayoría de profesores están de acuerdo en que no todas las CTs pueden ser evaluadas a través del TFG. Al mismo tiempo, se pone de relevancia el papel protagonista que los docentes otorgan al alumno al asignarle un número elevado de cuestiones sobre las que debería autoevaluarse.

Creemos que ésta es una línea de investigación que convendría explorar con mayor profundidad, analizando las diferencias que se puedan observar entre distintas titulaciones puesto que cada perfil profesional tendrá unos requerimientos diferentes. Así mismo, convendría ampliar el panel de expertos incluyendo a otros colectivos interesados como por ejemplo los colegios profesionales.

\section{Referencias}

DELBECQ A.(1989). Técnicas grupales para la planeación. México D.F: Editorial Trillas.

GALLEGO L., JULIA, J.F. (2003). Principios cooperativos y eficacia económica. Un análisis delphi en el contexto normativo español. Revista CIRIEC-ESPAÑA $N^{\circ} 44$, p. 231-259.

GUPTA U., CLARKE R. (1996). Theory and Applications of the Delphi Technique: A Bibliography (1975-1994). Technological Forecasting and Social Change 53 (2), p. 185-211.

LANDETA, J. (1999). El método Delphi. Una técnica de previsión para la incertidumbre. Barcelona: Ed. Ariel.

UNIVERSITAT POLITÈCNICA DE VALÈNCIA (2013). Competencias transversales. Manual de uso interno. 


\section{ANEXO I}

COMPETENCIAS TRANSVERSALES en el TFG - BIOTECNOLOGÍA

El objetivo de este cuestionario es seleccionar las competencias transversales UPV relacionadas con la titulación de Grado en Biotecnología y ver cuáles y cómo se pueden evaluar estas competencias en el Trabajo Fin de Grado (TFG).

La encuesta se realizará en dos iteraciones, mediante la aplicación del método Delphi.

Por favor, escribe tu dirección de correo electrónico *

Este dato es necesario para que te enviemos la segunda iteración del cuestionario

1.- El siguiente listado incluye las Competencias Transversales definidas por la UPV, por favor, señala cuál crees que es el grado de relevancia en la titulación.

\begin{tabular}{|l|l|l|l|l|l|}
\hline & $\begin{array}{l}\text { Nada } \\
\text { relevante }\end{array}$ & $\begin{array}{l}\text { Poco } \\
\text { relevante }\end{array}$ & $\begin{array}{l}\text { Término } \\
\text { medio }\end{array}$ & $\begin{array}{l}\text { Algo } \\
\text { relevante }\end{array}$ & $\begin{array}{l}\text { Muy } \\
\text { relevante }\end{array}$ \\
\hline $\begin{array}{l}\text { 1 COMPRENSIÓN E } \\
\text { INTEGRACIÓN: clasificar, } \\
\text { analizar y sintetizar. } \\
\text { Descubrir razones. }\end{array}$ & & & & & \\
\hline $\begin{array}{l}\text { 2 APLICACIÓN Y } \\
\text { PENSAMIENTO } \\
\text { PRÁCTICO: adaptarse a } \\
\text { nuevas situaciones, tomar } \\
\text { decisiones, enfrentarse a un } \\
\text { cierto tipo de incertidumbre. }\end{array}$ & & & & & \\
\hline $\begin{array}{l}\text { 3 ANÁLISIS Y } \\
\text { RESOLUCIÓN DE } \\
\text { PROBLEMAS. }\end{array}$ & & & & & \\
\hline $\begin{array}{l}\text { 4 INNOVACIÓN, } \\
\text { CREATIVIDAD Y } \\
\text { EMPRENDIMIENTO. }\end{array}$ & & & & & \\
\hline $\begin{array}{l}\text { 5 DISEÑO Y PROYECTO. } \\
\text { Trabajo realizado en tiempo } \\
\text { determinado con el objetivo } \\
\text { de diseñar un producto o } \\
\text { servicio. }\end{array}$ & & & & & \\
\hline 6 TRABAJO EN EQUIPO Y & & & & & \\
\hline
\end{tabular}

(cc) EY-NC-ND 2015, Universitat Politècnica de València

Congreso In-Red (2015) 


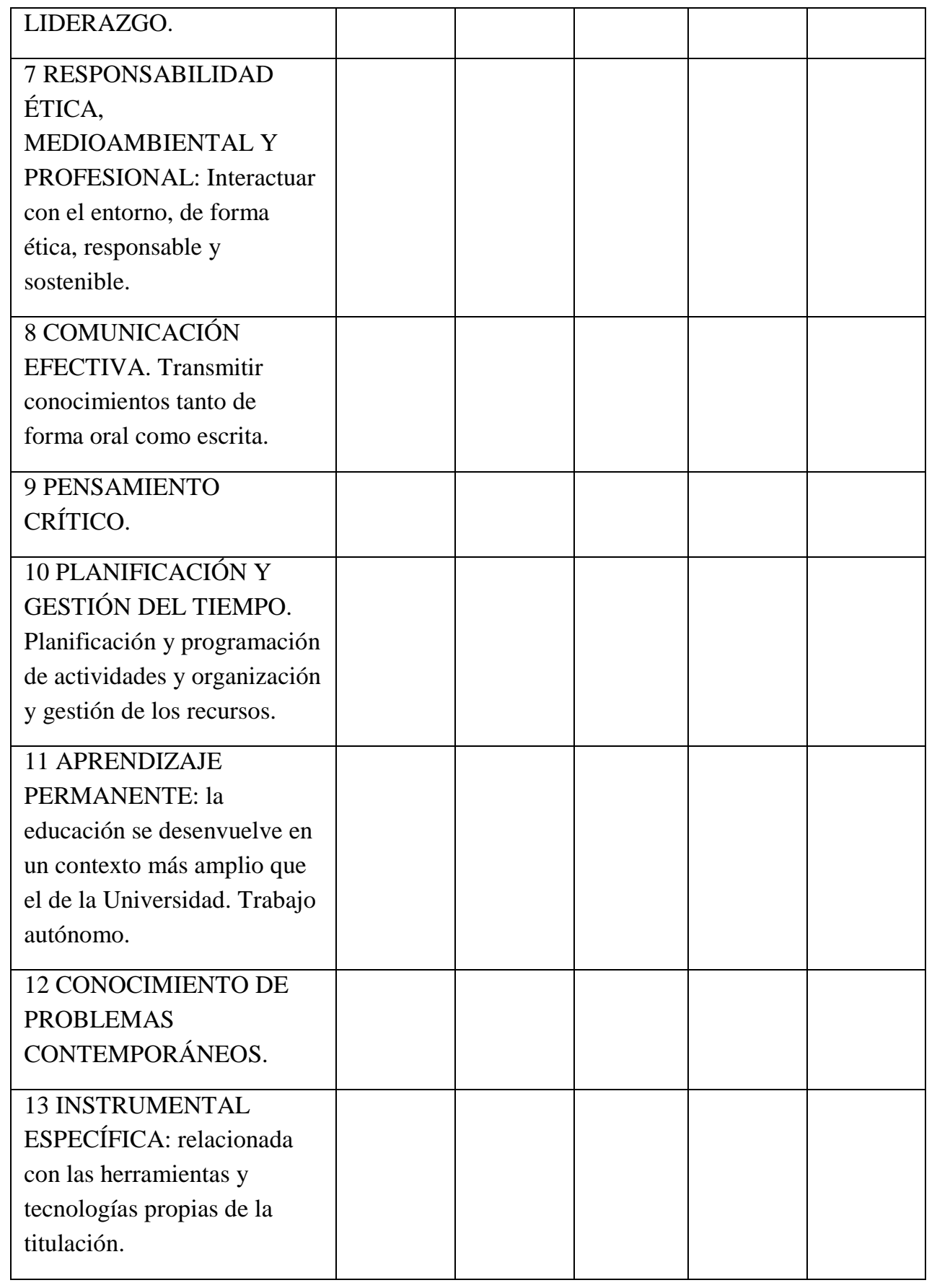

2.- De las competencias que son importantes para la titulación, si se considera su aplicación 
en el Trabajo Final de Grado (TFG), ¿quién consideras que podría valorarlas? Se puede señalar más de una opción.

1 COMPRENSIÓN E INTEGRACIÓN: clasificar, analizar y sintetizar. Descubrir razones.

$\square$ No es importante o no se puede evaluar en el TFG

$\square$ El tutor, mediante un informe

$\square$ La comisión de TFG, con el Trabajo presentado

$\square$ El tribunal, tras la sesión de defensa

$\square$ El alumno, con una autoevaluación

(y así sucesivamente con las 13 CTs) 